\title{
Needs and Enabling Technologies for Stretchable Electronics Commercialization
}

\section{Edward Tan ${ }^{1}$, Qingshen Jing ${ }^{2}$, Michael Smith², Sohini Kar-Narayan ${ }^{2}$ and Luigi Occhipinti ${ }^{1}$}

${ }^{1}$ Electrical Engineering Division, Department of Engineering, University of Cambridge, $9 \mathrm{JJ}$ Thomson Avenue, Cambridge, CB3 0FA, United Kingdom

${ }^{2}$ Department of Materials Science and Metallurgy, University of Cambridge, 27 Charles Babbage Road, Cambridge, CB3 0FS, United Kingdom

\begin{abstract}
$\underline{\text { Abstract }}$
Stretchable electronics represent an emerging class of devices that can be compressed, twisted and conform to very complicated shapes. The mechanical and electrical compliances of the technology promise to open up applications for healthcare, energy and entertainment purposes. However, advancement in the field has been hindered by material related constraints. Moreover, the current microfabrication facilities are optimized for rigid substrates such as silicon, which have significant different properties compared to elastomers. In this paper, four categories of enabling technologies for stretchable electronics commercialization are critically reviewed, namely: the novel design of stretchable structures, use of non-conventional materials, state-of-art printing techniques and also the patterning of electrodes or metal interconnects via conventional manufacturing techniques.
\end{abstract}

\section{$\underline{\text { Introduction }}$}

Multidisciplinary research has paved ways towards a new form of electronics which is not only flexible but also conformable and deformable; while ideally maintaining excellent electrical properties under strain. Known as 'stretchable electronics', new devices such as sensors, transistors and circuits, lighting elements, power sources, as well as their interconnections, exhibit closer resemblance to the human body than their rigid counterparts in terms of mechanical properties ${ }^{1,2,3}$. Thus, stretchable electronics can improve not only existing technologies but also enable a wide range of novel applications. For example, thin micro-electrodes made on soft and elastic substrate have been developed for neural prosthetics, neural signal recording and neuromodulation ${ }^{3,4}$. Compared to their rigid counterparts, these neural electrodes show improved mechanical compliance, offered better structural interface with the nervous system and are highly robust. Artificial skin have also been fabricated to detect external stimuli such as pressure, temperature, strain and humidity, which have huge implications for robotics and also amputated patients ${ }^{5}$. Other devices such as epidermal sensors for vital sign monitoring and stretchable energy harvesting for selfpowered electronics have also been shown ${ }^{6,7}$. More recently, stretchable electronics have found their ways into the entertainment realm. Kim et al. had made a highly stretchable touchpad that can be worn around the arm for new gaming experiences ${ }^{8}$.

Stretchable electronics are rapidly growing at a compound annual growth rate (CAGR) of $121.3 \%$, forecasted to reach $\$ 411.85 \mathrm{~m}$ in $2023^{9}$. However, when considering mechanically compliant smart patches and implantable electronic devices for both biosensing and drug delivery applications in personalized theranostic and stratified medicine platforms, market data are less clear. According to Future Markets, potential addressable market for the introduction of these technologies into commercial streams or emerging markets form an opportunity worth between $\$ 8.2 \mathrm{bn}$ and $\$ 16.2 \mathrm{bn}$, when penetration rates varying between $20 \%$ and $50 \%$ are considered, in: $i$ ) disposable medical sensor, ii) blood glucose monitoring, iii) 
cosmetic patches and $i v$ ) medical smart patches whose market introduction is expected by $2020^{10}$.

The technological hurdles that hinder the commercialization of stretchable electronics include the use of elastomeric materials and the fabrication processes of these devices. Considerable progress has been made to advance this field. In this paper, we cover some of the innovations that enable the development of these devices, which include the novel design of stretchable structures, use of non-conventional materials, state-of-art printing techniques and also the patterning of electrodes or metal interconnects via conventional manufacturing techniques.

\section{$\underline{\text { Stretchable Structures: Devices and Interconnects }}$}

Since early studies on the mechanical compliance of human skin, it has been clear that electronics integrated on the human skin must undergo strains of up to $30 \%$ without constraining natural motions ${ }^{11}$. Reaching this level of stretchability or higher also ensures that the elastic behaviour of human skin can be mimicked, so that a wearable device in form of electronic tattoo or smart patch can perform reliably when the substrate is subjected to the same level of strain. Moreover, implantable electronic devices designed to interact directly with organs, such as the heart muscle, the spinal cord or the brain, are often required to show the same mechanical compliance as the organ itself. This means that actual electronic interfaces need to be fabricated with biocompatible materials having the same values of Young modulus of the target organ. This in practice justifies the scientific and industrial interests in looking at soft electronic devices that can be stretched up to $100 \%$ or more of their initial dimensions within their elastic range, so that any changes in terms of their physical (i.e. mechanical, electrical, optical) characteristics are reversible under multiple cycles. Finally, sensors and electrodes on compliant or inflatable balloon catheters for minimally invasive surgical procedures require even higher levels of deformation, i.e. strains of well over $100 \%{ }^{12}$.

Stretchability can be achieved with conventional hard, rigid or brittle materials used in the electronic industry via novel device architectures or bespoke design of their interconnecting structures. In an early demonstration of the concept, Khang et al. had fabricated stretchable $\mathrm{Si}$ MOSFETs and $p-n$ diodes on thin silicon ribbons bonded to polydimethylsiloxane (PDMS) ${ }^{13}$. The multistep process first started with the patterning of silicon ribbons on a Si-on-insulator (SOI) wafer. These structures with only their two ends connected to the wafer were subsequently brought into contact with a pre-stretched PDMS. Upon release of the strain, the silicon formed into wavy structures with defined wavelength and amplitudes that accommodates deformation $^{14}$. In the study, devices maintained their properties even after 100 cycles of compression and stretching. Recent examples that utilize the same post-buckling strategy to create wavy structures include highly stretchable arrays of nanostructures and sheath core sheath-core fibers for superelastic electronics, sensors and muscles ${ }^{15,16}$.

However, as opposite to non-ceramic based materials that are commonly adopted in printed and flexible electronics, crystalline silicon, thinned down to just few microns in order to become flexible, shows several piezoeffects, both on passive and active devices. These include piezoresistivity in silicon-based resistors, piezojunction effects in bipolar transistors, current sensitivity to magnetic field (PiezoHall), piezotunneling in tunnelling diodes, and change in carrier mobility of MOSFET devices (piezoMOS) under bending ${ }^{17}$.

Another approach is to obtain stretchability by distributing active devices on rigid subcircuit islands over an elastomer as shown in figure $1^{18}$. Kim et al. had further built on this method by introducing out-of-plane arc shaped interconnects for islands of active devices ${ }^{19}$. This can be done by attaching only the device islands onto a pre-stretched PDMS with prior 
surface activation of the elastomer via oxygen plasma. Once the stress was released, the compressive forces caused the unattached interconnects to protrude and thus, forming arcshape structures which can be deformed. By introducing serpentine shapes, it was further shown by the authors that the electronic systems built on the PDMS can be strained up to $100 \%$ reversibly through this technique. When these structures are stretched within their limit, the electrical properties of the system approach its planar counterparts as the serpentine interconnects compensate the applied strained (stable up to 1,000 cycles) through the change in height and geometry.

Other structures that accommodate stretching include planar horseshoe interconnects ${ }^{20}$. Important design parameters include width of the track, radius of curvature and turning angle of the horseshoe tracks as shown in figure 2. More recently, Fan et al. had explored fractal design concepts such as Peano, Greek cross and Vicsek for stretchable electronics ${ }^{21}$. The advantage of these layouts includes the endurance to withstand biaxial, radial and other deformation mode.

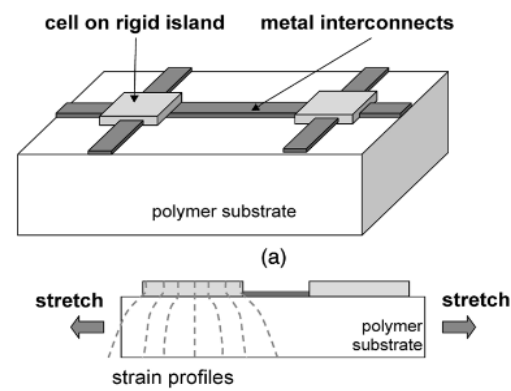

(b)

Figure 1. Stretchable device architecture on a polymer substrate (a) Active devices are fabricated on rigid subcircuit islands connected via stretchable interconnects (b) Cross-section of the electronic surface with strain profiles when stretched. Reproduced with permission from Ref ${ }^{18}$ Copyright 2005 IEEE.
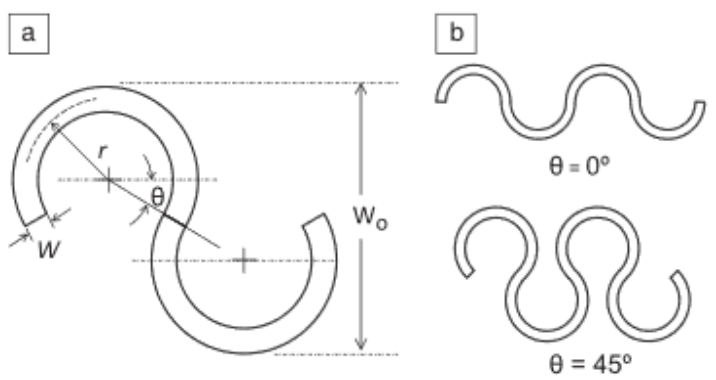

Figure 2. Stretchable horseshoe interconnect (a) geometrical parameters where $r=$ radius of curvature, $\theta=$ turning degrees, $\mathrm{W}=$ track width, $\mathrm{W}_{\mathrm{o}}=$ overall width. (b) horseshoe interconnect of different turning degrees. Reproduced with permission from Ref ${ }^{20}$ Copyright 2012 Cambridge University Press.

\section{Materials for Stretchable Electronics}

Due to the intrinsic low strain capability of conventional materials, alternative materials have been explored. Polymeric materials such as PDMS and polyurethane are commonly used as alternative substrates to silicon wafers ${ }^{22}$. While the majority of organic materials can generally be rendered flexible, such materials are not stretchable, which is a key mechanical property necessary to realize applications of E-skin for prosthetics, artificial intelligence, systems for robotics, personal health monitoring, biocompatibility, and communication devices. In order to overcome these limitations, intrinsically stretchable and self-healing polymeric materials for electronics, including conducting, dielectric and semiconducting polymer have been reported ${ }^{23,24}$. Researchers have also developed a wide range of conductors that are intrinsically deformable, including metal based, carbon nanocomposite based and conducting polymer based materials.

In the case of metal-based inks, for instance, film conductivity is given by their formulation, containing conductive material in form of micro or nanoparticles, a binder and solvent, so that conductive particles are bonded together to form a conductive film when the solvent leaves the ink after deposition, which rebuild the bonding at molecular and atomic levels ${ }^{25,26}$.Therefore, if the electrical performances of printed inks are nowadays approaching those of the same materials deposited with conventional methods, the electro-mechanical 
properties of materials deposited by printing might be substantially different. However, not all inks require the presence of binder and solvent. One such example the liquid metal alloy of gallium, indium and tin, known as 'Galinstan', that can be used directly as a conductive ink without requiring any solvent thanks to its low melting temperature at the eutectic composition $^{27}$. The fluidity may remain after the ink has been printed, which enhances the stretchability, but this does however require further packaging ${ }^{28}$.

Other promising composites have been reported with carbon-based inks. Gao et al. had printed pristine graphene ink which were still conductive after 1000 bending cycles under a folding angle of $180^{\circ} 29$. Highly conductive elastic conductors have been reported by Chun et $a l .{ }^{30}$. The metal and carbon nanotubes hybrid composites displayed a conductivity of $5710 \mathrm{~S}$ $\mathrm{cm}^{-1}$ and can be stretched up to $140 \%$ strain. Sekiguchi et al. had fabricated elastomeric field effect transistors based on single-walled carbon nanotubes which could be stretched (elasticity: $\sim 110 \%)$, bent, compressed $(>4.0 \mathrm{MPa}$, by a car and heels), impacted $(>6.26 \mathrm{~kg}$ $\mathrm{m} / \mathrm{s}$, by a hammer), and laundered ${ }^{31}$. Many of the inks need post-deposition treatment, such as sintering, in order to maximize their functionality ${ }^{25}$.

\section{Printing Technologies for Stretchable Electronics}

Printing technologies represent some of the enabling tools for the adoption of the aforementioned inks. Compared to conventional microfabrication processes, printing technologies are potentially cheaper and are able to deposit a wide range of both active and passive materials onto a generic planar or conformable substrate ${ }^{32}$. Three printing approaches: screen printing, inkjet printing and, more recently, aerosol jet printing is discussed here.

Screen printing is a well-established approach that utilizes a screen of woven material patterned with different sizes of the mesh and positioned at short distance from the substrate to be printed on. When ink is spread over the screen by squeegee, the ink is transferred from the screen to the substrate underneath according to the pattern contained in the screen ${ }^{33}$. One of the characteristics of screen printing process is related to direct contact of screen and substrate during printing, whose limitations include control of accuracy, especially on soft substrates, and potential contamination on bio-compatible substrate applications.

On the other hand, jet printing methods, also called "direct writing" provide non-contact printing condition that can minimize the stated issues. Thickness and resolution are two important aspects for high performance stretchable electrodes. It has been decades since the first inkjet printing technique was commercialised back in the seventies, and the technique has been diversely developed and well-established for precisely delivering tiny amount of liquid inks at relatively high resolution with accurate automation control. A common structure of the technique includes an inkjet printhead that has a small nozzle aperture on the tip, with a typical nozzle size of $10-50 \mu \mathrm{m}^{34}$. Ink is driven through the printhead from where it forms droplets $(20-100 \mu \mathrm{m})$ for deposition on the substrate. The process can be further assisted by heating, airflow, electric field or acoustic waves, to minimize the size of droplet formation ${ }^{35}$. The simple structure of the inkjet printhead allows researchers to fabricate proprietary printheads to meet special needs ${ }^{28}$. A wide range of materials can be formulated for inkjet printing, including metallic nanoparticle inks, carbon inks, polymers or inorganic particle inks. However, due to the direct contact between the ink and the nozzle, the tolerance of ink viscosity is often limited in the range $10-20 \mathrm{cP}^{34}$.

One of the potential application of stretchable electrode is on devices that conform reliably to human skin, providing optimal comfort and dynamic fit, as in the case of smart patches and electronic tattoos ${ }^{36}$. Thus, thinner electrodes and thin-film deposition methods on different substrates, such as thin elastomers and hydrocolloids help reduce the overall thickness of the 
stretchable device towards the so called "imperceptible electronics" 37 . In other circumstances where mechanical robustness is more important, for example in cloth, thicker electrodes are required. As a result, different printing technology may be called for different purpose. While inkjet printing is limited by relatively low ink viscosities, aerosol jet printing can tolerate a much larger viscosity range, between 1 and $1000 \mathrm{cP}$. Unlike in inkjet printing where ink droplets formed from printhead are individually placed on the substrate and coalesce to form continuity, aerosol printing forces the ink to form a fine mist, or "aerosol" that is further transferred to the substrate in a flow of carrier gas (e.g. nitrogen). Atomising transducer, based on either pneumatic force (Venturi effect) or ultrasonic energy, are used to generate aerosol of ink droplets typically 1 to $5 \mu \mathrm{m}$ in diameter. The mists are further surrounded by a guided flow of nitrogen carrier gas and fed towards the deposition head, where they are injected out in the presence of a second gas flow - the sheath gas (also nitrogen) that helps constrain the ink mists to a small area. The opening end of the tip is between $100-300 \mu \mathrm{m}$ in diameter, however, the action of the sheath gas enables focusing of the ink in an area as small as $10 \mu \mathrm{m}$ in diameter ${ }^{38}$. Advantages of this technique include highly customised features, choice of wide range of materials, large range of permissible ink viscosities, and reduction in material waste. Meanwhile, the presence of sheath gas flow additionally provides a cleaning mechanism of the substrate during the printing process. A wide range of materials including polymers, biological materials, carbon nanotubes and semiconducting materials have been reported successfully printed using aerosol jet printing technique $39,40,41,42$. Moreover, by combining two different atomisers operating in the same printing work, different inks can be mixed in the aerosol form, and load ration against each other can be adjusted to achieve ondemand functionalities ${ }^{43}$. Table 1 shows a comparison between the printing techniques reviewed.

Table 1 - Comparison of the basic characteristics between the three common techniques

\begin{tabular}{lllll}
\hline Technique & Nozzle $(\boldsymbol{\mu m})^{34,44}$ & $\begin{array}{l}\text { Ink Viscosity } \\
(\mathbf{c P})^{33,34,44}\end{array}$ & Resolution $(\boldsymbol{\mu m})^{33,44}$ & $\begin{array}{l}\text { Maximum } \\
\text { Speed }(\mathbf{m} / \mathbf{s})^{33,38}\end{array}$ \\
\hline Inkjet & $10-50$ & $10-20$ & $20-100$ & 0.2 \\
Screen & N/A & $\sim 100$ & $>100$ & 0.5 \\
Aerosol & $100-300$ & $0.7-1000$ & $5-30$ & 0.2 \\
\hline
\end{tabular}

\section{$\underline{\text { Repurposing conventional manufacturing techniques for stretchable electronics }}$}

Patterning thin film metal electrodes or interconnects on elastomeric substrates can be done via established fabrication techniques namely: lift-off, wet etching, shadow masking and pattern transfer. The main advantage of using these methods is related to the possibility of relatively quick industrialization pathways towards commercialization, together with the use of conventional, often depreciated manufacturing facilities. However, when the unique physical properties exhibited by elastomers are taken into account, such as the relatively large coefficient of thermal expansion (CTE) and swelling characteristics in commonly used solvents, major fabrication challenges are observed ${ }^{45,46,47,48}$.

Due to the significant mismatch in CTE between most common photoresists (PR) that are commercially available and the elastomeric PDMS substrate, cracks are generally formed since early photolithographic process steps, which pose serious concerns on the reliability and reproducibility of the method. In an attempt to mitigate the effects of PDMS expansion during baking steps, Patel et al. used an epoxy-based SU-8 as sacrificial mask for lift-off, as the negative resist is characterized by CTE values closer to $\mathrm{PDMS}^{49}$. By slowing the temperature ramping process, it was possible to avoid thermal cracking of the SU-8 mask. 
However, cured SU-8 is difficult to be removed by solvents, so mechanical peeling of the mask is required. This limits the range of patterns that can be obtained, and affects the overall yield and reliability. Guo et al. approached these issues with SU-8 lift-off obtained by introducing polyacrylic acid (PAA) as intermediate layer between the epoxy resist and $\mathrm{PDMS}^{45}$. PAA can be removed by water and also serves as barrier to limit solvents from reaching the bulk PDMS to minimize swelling ${ }^{48}$. Nevertheless, pattern distortions and microcracks were observed due to the large residual stress in the SU-8 film. Microcracks resulted from the differences in CTE remain unresolved to date based on known literatures. However, a recent work showed that these microcracks (typical width $<1 \mu \mathrm{m}$ ) do not present major concerns when patterning silver nanowires which are too large to penetrate through ${ }^{50}$

Examples of gold patterning fabricated via wet etching have been shown by several authors. Meacham et al. had constructed an elastic multi-electrode array based on PDMS with the method described above ${ }^{51}$. Gold was deposited on the PDMS, patterned through photolithography and subsequently etched by gold etchant. In a similar study, Lacour et al. introduced chromium stacks in between the PDMS and gold layer to improve the robustness as the transition metal is commonly used as adhesive layers ${ }^{46}$. However, the etching profile obtained by wet treatment typically result in undercut due isotropic nature of the chemical reactions ${ }^{52}$. An undercut is either advantageous due to the easy removal of resist at the end stage or undesirable as dense patterns might merge and supporting structure can collapse. More importantly, etchants used in the processes are typically toxic which can be a critical issue for biocompatible applications if not sterilized properly. Wet etching of electrodes is now rarely seen as means to fabricate electrodes on elastomers.

On the other hand, stencil technology provides an alternative way to pattern metal electrodes on elastomeric substrates. It involves placing a sheet of pre-defined pattern in front of the substrate, which allows metal vapour to only pass through the pre-defined patterns. Minev et al. had patterned gold on PDMS via a Kapton ${ }^{\oplus}$ shadow mask $^{53}$. Similarly, Gong et al. deposited gold and titanium films through a shadow mask to fabricate interdigitated electrodes ${ }^{54}$. However, the main limitation of this method is the relatively low resolution $(>100 \mu \mathrm{m})$ achievable which can potentially hinder the technological progress especially in imaging and recording systems based on stretchable materials ${ }^{55,56}$.

More recently, researchers have developed techniques based on pattern transfer which not only mitigated the stated problems in lift-off but also avoided the direct exposure of elastomers to toxic chemicals. For example, Byun et al. had devised a method which first involve fabricating electrodes on silicon via standard microfabrication techniques and subsequently transfer the patterns to PDMS via (3-mercaptopropyl)trimethoxysilane as adhesive $^{57}$. In another approach, Deng et al. had also transferred electrodes made on a rigid substrate through carrier substrates such as poly(methylmethacrylate) ${ }^{58}$. The downsides of these methods typically include the additional processing steps required, difficulties when handling the carrier substrates and also loss of patterns during transfer step(s).

\section{Conclusions}

Electronics that are highly stretchable and conductive have been demonstrated through efforts in material innovations and design, supported by both existing microfabrication technologies and also printing techniques. The field continues to attract strong interest from both the scientific and business community due to unmet needs that remain unsolved by conventional, silicon-based, electronic manufacturing and materials used by semiconductor industry. In this paper, we reviewed the enabling technologies required for stretchable electronics that are compliant with industry standards in terms of yield (90\%), mechanical robustness (under repeated bending and stretching cycles, e.g. 10000-100000 cycles with less 
than 5\% degradation in performance) and reliability (i.e. under environmental and operational stress), as needed to enable commercialization of high-end applications that are at the frontier between bio-electronics and healthcare. Examples of such applications include smart prosthetic skin, implantable organ interfaces, "soft robotics" and "imperceptible electronics" where mechanical compliance is a key requirement in order to help the electronic system interact more naturally with living systems that are intrinsically soft and malleable. Using the enabling technologies discussed in this review, both emerging and existing electronic device manufacturers and system integrators are in the position to develop and commercialize new classes of products that address the reported manufacturing challenges of stretchable electronics, as required by applications in the energy, medical and military realm.

\section{Acknowledgements}

E.T is grateful for the studentship funding from Trinity Hall, University of Cambridge. E.T and L.O acknowledge funding support from the UK Engineering and Physical Sciences Research Council through the EPSRC Centre for Innovative Manufacturing in Large Area Electronics (Grant no. EP/K03099X/1). S.K-N is grateful for financial support from the European Research Council through an ERC Starting Grant (Grant no. ERC-2014-STG639526, NANOGEN). Q.J is grateful for financial support through a Marie Sklodowska Curie Fellowship, H2020-MSCAIF-2015-702868. M.S is grateful for studentship funding from the Cambridge Trust.

\section{$\underline{\text { References }}$}

1. Chortos, A. \& Bao, Z. Skin-inspired electronic devices. Mater. Today 17, 321-331 (2014).

2. Kim, D.-H., Ghaffari, R., Lu, N. \& Rogers, J. A. Flexible and Stretchable Electronics for Biointegrated Devices. Annu. Rev. Biomed. Eng. 14, 113-128 (2012).

3. Lacour, S. P. et al. Flexible and stretchable micro-electrodes for in vitro and in vivo neural interfaces. Med Biol Eng Comput 48, 945-54 (2010).

4. Lee, J. H., Kim, H., Kim, J. H. \& Lee, S.-H. Soft implantable microelectrodes for future medicine: prosthetics, neural signal recording and neuromodulation. Lab Chip 16, 959-976 (2016).

5. Chortos, A., Liu, J. \& Bao, Z. Pursuing prosthetic electronic skin. Nat. Mater. 15, 937-950 (2016).

6. Liu, Y. et al. Epidermal mechano-acoustic sensing electronics for cardiovascular diagnostics and human-machine interfaces. Sci. Adv. 2, 1-12 (2016).

7. Fan, F. R., Tang, W. \& Wang, Z. L. Flexible Nanogenerators for Energy Harvesting and SelfPowered Electronics. Adv. Mater. 28, 4283-4305 (2016).

8. Kim, C.-C. et al. Highly stretchable, transparent ionic touch panel. Science 353, 682-7 (2016).

9. Marketsandmarkets.com. Stretchable Electronics Market by Component - 2023. (2015). Available at: http://www.marketsandmarkets.com/Market-Reports/stretchable-electronicmarket-181339852.html. (Accessed: 6th December 2016)

10. Future Markets, inc. Nanotechnology in Smart Textiles and Wearables, Medical and Healthcare. (2016). Available at: http://www.reportlinker.com/p04422604summary/Nanotechnology-in-Smart-Textiles-and-Wearables.html.(Accessed: 6th December 2016)

11. Pailler-Mattei, C., Bec, S. \& Zahouani, H. In vivo measurements of the elastic mechanical properties of human skin by indentation tests. Med. Eng. Phys. 30, 599-606 (2008).

12. Kim, D.-H. et al. Materials for multifunctional balloon catheters with capabilities in cardiac electrophysiological mapping and ablation therapy. Nat. Mater. 10, 316-23 (2011).

13. Khang, D.-Y., Jiang, H., Huang, Y. \& Rogers, J. A. A Stretchable Form of Single-Crystal Silicon for High-Performance Electronics on Rubber Substrates. Science 311, 208-212 (2006).

14. Kim, J. et al. Stretchable silicon nanoribbon electronics for skin prosthesis. Nat. Commun. 5, 
5747 (2014).

15. Gao, L. et al. Optics and Nonlinear Buckling Mechanics in Large-Area, Highly Stretchable Arrays of Plasmonic Nanostructures. ACS Nano 9, 5968-5975 (2015).

16. Liu, Z. F. et al. Hierarchically buckled sheath-core fibers for superelastic electronics, sensors, and muscles. Science. 349, (2015).

17. Fruett, F. The Piezojunction Effect in Silicon, its Consequences and Applications for Integrated Circuits and Sensors. (Delft University Press, Delft, 2001).

18. Lacour, S. P., Jones, J., Wagner, S., Li, T. \& Suo, Z. Stretchable Interconnects for Elastic Electronic Surfaces. Proceedings of the IEEE 93, 1459-1466 (2005).

19. Kim, D.-H. et al. Materials and noncoplanar mesh designs for integrated circuits with linear elastic responses to extreme mechanical deformations. Proc. Natl. Acad. Sci. U. S. A. 105, 18675-80 (2008).

20. Vanfleteren, J. et al. Printed circuit board technology inspired stretchable circuits. MRS Bull. 37, 254-260 (2012).

21. Fan, J. A. et al. Fractal design concepts for stretchable electronics. Nat. Commun. 5, 3266 (2014).

22. T. Someya, editor, Stretchable Electronics (WILEY-VCH Verlag GmbH \& Co. KGaA, Weinheim, 2013).

23. Young Oh, J. et al. Intrinsically stretchable and healable semiconducting polymer for organic transistors. Nat. Publ. Gr. 539, (2016).

24. Benight, S. J., Wang, C., Tok, J. B. H. \& Bao, Z. Stretchable and self-healing polymers and devices for electronic skin. Progress in Polymer Science 38, 1961-1977 (2013).

25. Mohammed, A. \& Pecht, M. A stretchable and screen-printable conductive ink for stretchable electronics. Appl. Phys. Lett. 109, 184101 (2016).

26. Matsuhisa, N. et al. Printable elastic conductors with a high conductivity for electronic textile applications. Nat. Commun. 6, 7461 (2015).

27. Jin, S. W., Park, J., Hong, S. Y., Park, H. \& Jeong, Y. R. Stretchable Loudspeaker using Liquid Metal Microchannel. Nat. Publ. Gr. 5, 1-13 (2000).

28. Li, G., Wu, X. \& Lee, D.-W. A galinstan-based inkjet printing system for highly stretchable electronics with self-healing capability. Lab Chip 16, 1366-1373 (2016).

29. Gao, Y., Shi, W., Wang, W., Leng, Y. \& Zhao, Y. Inkjet Printing Patterns of Highly Conductive Pristine Graphene on Flexible Substrates. Ind. Eng. Chem. Res. 53, 16777-16784 (2014).

30. Chun, K.-Y. et al. Highly conductive, printable and stretchable composite films of carbon nanotubes and silver. Nat. Nanotechnol. 5, 853-857 (2010).

31. Sekiguchi, A. et al. Robust and Soft Elastomeric Electronics Tolerant to Our Daily Lives. Nano Lett. 15, 5716-5723 (2015).

32. Seifert, T. et al. Additive Manufacturing Technologies Compared: Morphology of Deposits of Silver Ink Using Inkjet and Aerosol Jet Printing. Ind. Eng. Chem. Res. 54, 769-779 (2015).

33. Krebs, F. C. Fabrication and processing of polymer solar cells: A review of printing and coating techniques. Solar Energy Materials and Solar Cells 93, 394-412 (2009).

34. Tekin, E., Smith, P. J. \& Schubert, U. S. Inkjet printing as a deposition and patterning tool for polymers and inorganic particles. Soft Matter 4, 703-713 (2008).

35. Castrejón-Pita, J. R. et al. Future, opportunities and challenges of inkjet technologies. At. Sprays 23, 541-565 (2013).

36. Kim, J. et al. Highly Transparent and Stretchable Field-Effect Transistor Sensors Using Graphene-Nanowire Hybrid Nanostructures. Adv. Mater. 27, 3292-3297 (2015).

37. Kaltenbrunner, M. et al. An ultra-lightweight design for imperceptible plastic electronics. Nature 499, 458-463 (2013).

38. Optomec. Aerosol Jet 300 Series Systems - Datasheet. (2015). Available at: http://www.optomec.com/wp-content/uploads/2014/04/AJ-300-Datasheet_Web.pdf. (Accessed: 4th August 2016)

39. Cho, J. H. et al. Printable ion-gel gate dielectrics for low-voltage polymer thin-film transistors on plastic. Nat. Mater. 7, 900-906 (2008).

40. Grunwald, I. et al. Surface biofunctionalization and production of miniaturized sensor structures using aerosol printing technologies. Biofabrication 2, 014106 (2010). 
41. Ha, M. et al. Aerosol jet printed, low voltage, electrolyte gated carbon nanotube ring oscillators with sub-5 $\mu$ s stage delays. Nano Lett. 13, 954-960 (2013).

42. Tait, J. G. et al. Uniform Aerosol Jet printed polymer lines with $30 \mu \mathrm{m}$ width for $140 \mathrm{ppi}$ resolution RGB organic light emitting diodes. Org. Electron. physics, Mater. Appl. 22, 40-43 (2015).

43. Wang, K., Chang, Y. H., Zhang, C. \& Wang, B. Conductive-on-demand: Tailorable polyimide/carbon nanotube nanocomposite thin film by dual-material aerosol jet printing. Carbon N. Y. 98, 397-403 (2016).

44. Liu, R. et al. Fabrication of platinum-decorated single-walled carbon nanotube based hydrogen sensors by aerosol jet printing. Nanotechnology 23, 505301 (2012).

45. Guo, L. \& Deweerth, S. P. An effective lift-off method for patterning high-density gold interconnects on an elastomeric substrate. Small 6, 2847-2852 (2010).

46. Adrega, T. \& Lacour, S. P. Stretchable gold conductors embedded in PDMS and patterned by photolithography: fabrication and electromechanical characterization. J. Micromechanics Microengineering 20, 055025 (2010).

47. Chou, N. et al. Crack-free and reliable lithographical patterning methods on PDMS substrate. J. Micromechanics Microengineering 23, 125035 (2013).

48. Lee, J. N., Park, C. \& Whitesides, G. M. Solvent Compatibility of Poly(dimethylsiloxane)Based Microfluidic Devices. Anal. Chem. 75, 6544-6554 (2003).

49. Patel, J. N., Kaminska, B., Gray, B. L. \& Gates, B. D. A sacrificial SU-8 mask for direct metallization on PDMS. J. Micromechanics Microengineering 19, 115014 (2009).

50. Jeong, D.-W., Jang, N.-S., Kim, K.-H. \& Kim, J.-M. A stretchable sensor platform based on simple and scalable lift-off micropatterning of metal nanowire network. RSC Adv. 6, 7441874425 (2016).

51. Meacham, K. W., Giuly, R. J., Guo, L., Hochman, S. \& DeWeerth, S. P. A lithographicallypatterned, elastic multi-electrode array for surface stimulation of the spinal cord. Biomed. Microdevices 10, 259-269 (2008).

52. Franssila, S. Introduction to Microfabrication Introduction to Microfabrication Second Edition. (John Wiley \& Sons, Ltd, Chichester, 2010).

53. Minev, I. R. et al. Electronic dura mater for long-term multimodal neural interfaces. Science. 347, (2015).

54. Gong, S. et al. A wearable and highly sensitive pressure sensor with ultrathin gold nanowires. Nat. Commun. 5, 838-843 (2014).

55. Viventi, J. et al. Flexible, foldable, actively multiplexed, high-density electrode array for mapping brain activity in vivo. Nat. Neurosci. 14, 1599-605 (2011).

56. Song, Y. M. et al. Digital cameras with designs inspired by the arthropod eye. Nature 497, 9599 (2013).

57. Byun, I., Coleman, A. W. \& Kim, B. Transfer of thin Au films to polydimethylsiloxane (PDMS) with reliable bonding using (3-mercaptopropyl)trimethoxysilane (MPTMS) as a molecular adhesive. J. Micromech. Microeng 23, 85016-10 (2013).

58. Deng, W. et al. A High-yield Two-step Transfer Printing Method for Large-scale Fabrication of Organic Single-crystal Devices on Arbitrary Substrates. Sci. Rep. 4, 4772-4776 (2014). 